\title{
Editorial Nutritional Modulators in Chronic Heart Failure
}

\author{
Ram B. Singh ${ }^{1, *}$, Krasimira Hristova ${ }^{2}$, Galal El-Kilany ${ }^{3}$, Takahashi Toru ${ }^{4}$, Abdullah Shehab ${ }^{5}$, \\ Hilton Chaves ${ }^{6}$, Douglas W. Wilson ${ }^{7}$, Divya Rupini ${ }^{1}$, Neelesh Gupta ${ }^{8}$ and Rajeev Gupta ${ }^{9}$
}

\author{
${ }^{1}$ Halberg Hospital and Research Institute, Moradabad, India \\ ${ }^{2}$ National Heart Hospital, Sofia, Bulgaria \\ ${ }^{3}$ Dibba Hospital, Fujareh, UAE \\ ${ }^{4}$ Graduate School of Human Environmental Medicine, Fukuoka Women's University, Fukuoka, Japan \\ ${ }^{5} U A E$ University, $U A E$ \\ ${ }^{6}$ Federal University of Pernambuco, Recefe, Brazil \\ ${ }^{7}$ Formerly,Durham University, UK \\ ${ }^{8}$ Neelesh Gupta, Medical Student(Intern), Kasturba Hospital, BHEL, Bhopal, M.P., India \\ ${ }^{9}$ Kalba Hospital, Kalba, UAE
}

Chronic Heart failure (HF) may be associated with cardiac cachexia characterized with weight loss as well as with nutritional deficiency [1-4]. The increased oxidative stress in chronic HF attempts to consume various nutrients present in the cardiomyocyte as well as endogenous antioxidants. Vitamin $\mathrm{C}, \mathrm{E}$ and beta carotene, as well as coenzyme Q10 are potential antioxidants for protection against oxidative stress developed in HF patients [1-5]. The increase in homocysteine due to oxidative stress, is antagonized by vitamins; B6, B12 and folic acid. The decrease in magnesium and potassium due to long term diuretic therapy in HF which can predispose arrhythmiogenesis, leading to rhythm disturbances and sudden cardiac death [3-5]. L-carnitine, cysteins and taurine amino acids are also reduced in chronic $\mathrm{HF}$ and their supplementation may be protective [6-8]. It seems that these nutrients can be administered as nutraceuticals among these patients with chronic HF.

Apart from above micronutrients, calcium, selenium, zinc, thiamine, vitamin D and omega-3 fatty acids may play a more important role than previously thought [1-9]. Increased concentration of aldosterone and chronic use of loop diuretics increase urinary excretion of calcium and magnesium, leading to arrhythmias and secondary hyperparathyroidism, which in turn depletes calcium from bone and drives calcium into cells, causing further increase in oxidative stress [8-12]. Vitamin D deficiency, can enhance the damage, which now appears ubiquitous in northern

*Address correspondence to the author at the Halberg Hospital and Research Institute, Civil Lines, Moradabad (UP)244001, India;

E-mail: rbs@tsimtsoum.net latitudes. It is possible that supplementation of nutraceuticals and nutrient rich Mediterranean style diets may be protective against chronic HF [10-12].

\section{MECHANISMS OF NUTRIENT DEFICIENCY}

Clinical and experimental studies have demonstrated that following an injury to the cardiomyocyte during $\mathrm{HF}$, an intense inflammatory response occurs and leads to further injury and progression of the cardiac dilatation and dysfunction in conjunction with nutrient deficiency [13-16]. The released cell debris such as extracellular ATP, during tissue injury induces conformational changes in the components of the inflammation; such as the cryopyrin NLRP3 which is a sensor protein and the (Associated SpeckLike Protein Containing CARD (ASC); adaptor protein and triggers activation of caspase-1 and effector protein which are proinflammatory [15]. The underlying mechanism for nutritional deficiency as well as for clinical and biochemical manifestations of HF may be due in part, to neuro-humoral adaptations, such as activation of the renin-angiotensinaldosterone and sympathetic nervous systems by the lowoutput state, which can contribute to the maintenance of perfusion of vital organs. In $\mathrm{HF}$, catecholamine-stimulated contractility and increased heart rate can worsen coronary ischemia and heart rate variability resulting into arrhythmias and sudden cardiac death. Catecholamines, cortisol and angiotensin II, increase the free radical generation leading to oxidative stress which promotes the loss of myocytes by apoptosis [15, 16]. These mechanisms also induce maladaptive fetal isoforms of proteins involved in contraction, and hypertrophy, which might become worst in the presence of melatonin and nutrient deficiency [13-16]. 
The increase in plasma angiotensin II enhances angiotensin II signaling which further enhances sympathetic nerve activity through actions on both central and peripheral sites during chronic HF. Angiotensin II signaling is enhanced in different brain sites such as the paraventricular nucleus, the rostral ventrolateral medulla and the area postrema leading to neuronal deficiency of omega-3 fatty acids, flavonoids and coenzyme Q10 which are rich in the cell membranes [11, 13, 14]. Nutrient deficiency can cause increased adverse effects on these structures resulting into further imbalance in autonomic nervous system dysfunction, predisposing increased morbidity and mortality in chronic HF [13, 14]. In chronic HF, in peripheral regions, angiotensin II elevates both norepinephrine release and synthesis and inhibits norepinephrine uptake at nerve endings, which may contribute to the increase in sympathetic nerve activity and oxidative stress causing nutrient deficiency $[15,16]$. It is possible that increased circulating angiotensin II can directly act on the central nervous system via the subfornical organ and the area postrema to increase sympathetic outflow. Inhibition of angiotensin II formation and its type 1 receptor has been shown to have beneficial effects in patients with chronic HF. It is possible that blocking angiotensin II type 1 receptors can decrease sympathetic nerve activity and cardiac sympathetic afferent reflex when therapy is administered to the paraventricular nucleus $[14,15]$. Such therapy may be either pharmacological or nonpharmacological. In addition to cytokines, the natriuretic peptides appear to be the most popular of the biomarkers to indicate severity and prognosis; either brain natriuretic peptide (BNP) or NT-proBNP [N-terminal fragment BNP]. The values of these differ; BNP usually runs in lower numbers and NTproBNP in higher numbers and majority of the drugs used for the treatment of HF lower NT-proBNP $[4,15]$. Betablockers, angiotensin-converting enzyme (ACE) inhibitors, angiotensin receptor blockers, spironolactone, coenzyme Q10 and diuretics all lower NT-proBNP. The sensing of danger signals by cryopyrin, the activation of caspase-1 and the processing and release of interleukin-1 $\beta$ (IL-1 $\beta$ ) are all critical and modifiable steps in this process which worsen due to neuro-humoral dysfunction $[15,16]$. This biochemical process of HF may further worsen if there is coexisting deficiency of magnesium, coenzyme Q10, omega3 fatty acids, flavonoids, L-carnitine, taurine, antioxidants and vitamin B1 which appear to be important in cardiomyocyte remodeling [1-9] (Table 1).

\section{CLINICAL EVIDENCE}

The potential role of nutrition in the modulation of autonomic nervous system in the management of HF appears to be very interesting. In HF, autonomic imbalance causing receptor alterations, may have profound effects on cardiomyocyte biology and function. Inhibition of the sympathetic drive to the heart through $\beta$-receptor blockade and increase in parasympathetic activity via vagal stimulation has become a standard component of therapy for HF with a dilated left ventricle because of their effectiveness in inhibiting the ventricular structural remodeling process and in prolonging life $[16,17]$. HF is the leading cause of hospitalization and deaths in all the countries of the world and cellular deficiency of nutrients appears to be important in these deaths, particularly during cardiac cachexia [1-9]. A recent study reported increase in inflammatory mediators; IL-G and TNF-alpha among 116 patients of HF indicating severity and prognosis among these patients [14]. In a randomized, controlled trial, among 62 patients with CHF with lower Co Q10 levels and low ejection fraction, it was reported for the first time that, treatment with $\mathrm{CoQ} 10+\mathrm{L}-$ carnitine for 12 weeks can cause a marked reduction in IL-6 and TNF-alpha in the intervention group without such changes in the control group $(7.6 \pm 1.5 v s .11 .4 \pm 2.5 \mathrm{pg} / \mathrm{ml}$, $\mathrm{p}<0.01$ ) [7]. A twin study, comprising of 276 middle aged male twins, all twins underwent 24-hour ambulatory electrocardiogram (ECG) recording and fruit, vegetable and legume and nuts intake by food frequency questionnaire [17]. In all the subjects, time and frequency domain measures of HRV were calculated. After adjusting for energy intake, other nutritional factors, shared genes, and common environment, a 1-unit higher score for Mediterranean food intake was significantly associated with $3.9 \%$ to $13 \%$ higher time and frequency domain HRV parameters. Further

Table 1. Nutritional mediators in congestive heart failure.

\begin{tabular}{|l|l|}
\hline \multicolumn{1}{|c|}{ Studies } & Nutrient \\
\hline \hline Kumar et al, Acta Cardiol 2007(12) & Coenzyme Q10 \\
\hline Muzaffarian et al, Ann Intern Med 2011(22) & Omega-3 fatty acids \\
\hline Singh et al, Brit Heart J, Amer Heart J 1976(6,7) & Magnesium \\
\hline Singh et al, Amer Heart J,1976,1982(8) & Potassium \\
\hline Ito et al, Amino acids 2014;46:111-19 (6) & Taurine \\
\hline Kumar et al, Acta Cardiol 2007(12) & L-carnitine \\
\hline Witte et al 2001 (1) & B1 and other B vitamins \\
\hline Singh et al, Nutr Res, 2014(submitted) & Vitamines A,E, beta carotine \\
\hline Krasimira et al 2012 (16), Pittler et al, 2003(23) & Flavonoids \\
\hline Witte et al, JACC 2001;37:1765-74.(1) & Endogenous antioxidants, SODM, catalase, ceruloplasmin \\
\hline
\end{tabular}


controlling for known cardiovascular risk factors and use of fish oil supplements and medications did not substantially change the estimates, indicating that Mediterranean dietary pattern may be associated with higher HRV [17]. The effect of an alpha-linolenic acid and flavonoid rich diet on the circadian rhythm of cardiac events was studied among 1000 high risk patients of CVDs [18]. There was significant decline in the cardiac events in the second quarter of 24-hour scale in the intervention group compared to control group, indicating that such diets can also act on suprachiasmatic circadian clock. Flavonoids appear to have independent beneficial effects on vascular, cardiac and neuronal functions [19]. Plasma phospholipid fatty acid concentrations and other cardiovascular risk factors were measured in 2735 subjects in a cohort study. Plasma phospholipid EPA concentration was inversely associated with incident CHF; risk was approximately 50\% lower in the highest versus the lowest quartile (hazard ratio [HR], 0.52 [95\% CI, 0.38 to 0.72]; $\mathrm{P}$ for trend $=0.001$ ) [11]. Hawthorne extract which is rich in flavonoids has also been successfully used for treating chronic heart failure [12].

A randomized trial examined the effect of a combination of micronutrient supplementation on left ventricular function and quality of life in 30 elderly patients with stable systolic dysfunction (ejection fraction $<35 \%$ ) due to coronary disease [10]. The patients were randomized to receive a combination of calcium, magnesium, zinc, copper, selenium, vitamins $\mathrm{A}, \mathrm{C}, \mathrm{D}, \mathrm{E}, \mathrm{B} 6$ and $\mathrm{B} 12$, thiamine, riboflavin, folate, and coenzyme Q or placebo. After a follow up of 9 months, the treated group had reduced left ventricular volumes, increased ejection fraction $(+5 \% \mathrm{p}<0.05)$ and improved quality of life scores without any alteration in the control group. The intervention contained 10 different micronutrients at doses ranging from $1 / 3$ to 200 times the recommended daily intake. This small study offers some guidance as to which of these nutrients is essential or in what doses they should be given, but it does suggest the need for further investigation in this area. It is known that coenzyme Q10, omega-3 fatty acids and flavonoids work by activating nitric oxide release from the endothelium [1-12].

In patients with heart failure (HF), essential and non essential amino acid availability is declined, leading to abnormalities in cardiac and skeletal muscle metabolism, and eventually to a reduction in functional capacity and quality of life [6]. A prospective, open study evaluated the efficacy of the administration of a mixture of essential and semiessential amino acids in 13 patients with stable chronic HF. Patients received for three months a mixture of essential and semi-essential AAs (L-leucine, L-lysine, L-isoleucine, Lvaline, L-threonine, L-cystine, L-hystidine, Lphenylalanine), thiamine, pyridoxine at the dose of $4 \mathrm{~g}$ twice a day. The primary endpoint was to evaluate the effects of AAs supplementation on exercise tolerance at cardiopulmonary stress test and 6-minute walk test (6MWT). Patients were evaluated at baseline and after a three months follow-up. Compared with baseline, peak VO2 (baseline $14.8 \pm 3.9 \mathrm{~mL} /$ minute $/ \mathrm{kg}$ vs follow-up $16.8 \pm 5.1$ $\mathrm{mL} /$ minute $/ \mathrm{kg} ; \mathrm{P}=0.008$ ) and $\mathrm{VO} 2$ at anaerobic threshold improved significantly (baseline $9.0 \pm 3.8 \mathrm{~mL} /$ minute $/ \mathrm{kg} v \mathrm{~s}$ follow-up $12.4 \pm 3.9 \mathrm{~mL} /$ minute/ $\mathrm{kg} ; \mathrm{P}=0.002)$. Also the 6MWT distance increased at three months (baseline $439.1 \pm$
$64.3 \mathrm{~m} v$ s followup $474.2 \pm 89.0 \mathrm{~m} ; \mathrm{P}=0.006)$. A trend in the reduction of end systolic volume index (EDVI) (baseline $107.2 \pm 34.6 \mathrm{~mL} v s$ follow-up $99.2 \pm 34.3 \mathrm{~mm} ; \mathrm{P}=0.071$ ) was also observed.The study revealed that treatment with amino acids was safe, in patients with chronic HF and improved exercise tolerance but did not alter quality of life.

A randomized, controlled intervention trial examined the role of Mediterranean style, low caloric diet among 204 intervention group and 202 control group patients with recent myocardial infarction [20]. After one year of follow up, there was a significantly lower patients with left ventricular strain $(\mathrm{n}=19$ vs 34,9 vs $16.8 \%, \mathrm{p}<0.01)$, left ventricular hypertrophy $(\mathrm{n}=11$ vs 24,5 vs $17 \%, \mathrm{p}<0.01)$ and NYHA class III and IV HF ( $\mathrm{n}=8$ vs 14,4 vs $7 \%, \mathrm{p}<0.05)$. Combined left ventricular hypertrophy and class III and IV heart failure $(\mathrm{n}=19$ vs $42 ; 9.3$ vs $20.8 \%, \mathrm{P}<0.001)$ were significantly lower among patients receiving low caloric Mediterranean style diet compared to control group respectively. Ventricular ectopics were also significantly lower in the low energy experimental group compared to National Cholesterol Education Program (NCEP) low fat diet group ( $\mathrm{n}=17$ vs 60, 8 vs 30\%, $\mathrm{P}<0.001)$. At baseline, all these makers were comparable in the two groups. The IndoMediterranean diet heart study, randomized 499 patients in the intervention group and 501 in the control group who were at high risk of cardiovascular disease (CVDs) [21]. After a follow up of two years, assessment of dietary intakes revealed that intervention group was receiving significantly greater amount of fruits, vegetables, legumes, walnuts and almonds as well as mustard oil which is rich in omega-3 fatty acids. Cardiac complications such as left ventricular hypertrophy ( $\mathrm{n}=25$ vs 55,5 vs $11 \%, \mathrm{P}=0.0004)$, NYAH failure class III and IV ( $\mathrm{n}=11$ vs 35, 2.2 vs 7\%, $\mathrm{P}=0.0003$ ) were significantly lower in the intervention group compared to control. However, at baseline, these complications were similar in the two groups. In both trials, total cardiac events were significantly lower in the intervention group compared to control group. The Mediterranean style diets are rich sources of omega-3 fatty acids, flavonoids and essential and non essential amino acids which are known to have beneficial effects on neurons autonomic function [17]. These studies indicate that there is sufficient evidence to support that a large scale trial with multiple nutrients or Mediterranean style diet should be conducted to demonstrate their role in the management of autonomic dysfunction in patients with HF.

Nutrient deficiency has been observed among patients of hypertension, coronary artery disease, rheumatic heart disease, dyslipidemia, diabetes, obesity, genetic predisposition, viruses, and toxins which are major risk factors for HF [3-7]. The HF is characterized by evidence of cardiac systolic and/or diastolic dysfunction due to severe neurohumoral dysfunction and clinical signs of dyspnoea, palpitation, fatigue, and fluid retention in association with nutrient deficiency [4-7].

A recent study; PARADIGM-HF randomized > 8000 patients with depressed LV systolic function, almost all of them were in NYHA class 2-3, to treatment with LCZ696 or enalapril on top of other evidence-based therapies [22]. Patients who received the novel agent compared with the 
enalaprill group, showed 20\% drops in cardiovascular death or HF hospitalization and in cardiovascular death as a solo end point ( $p<0.001$ for both) over two to three years. Allcause mortality also declined; $0.80 \%(\mathrm{p}<0.001)$. Beyond this super angiotensin receptor-neprilysin inhibitor (ARNI), it is possible that repair of nutrient deficiencies by supplementation of CoQ10, L-carnitine, taurine, omega-3 fatty acids as well as B1, antioxidant flavonoids, might further improve the prognosis among patients with HF [112]. It seems that ARNI therapy combined with nutraceuticals or Mediterranean style diet, may be an additional approach for providing greater benefit because, repair of nutrient metabolism in the cardiomyocyte appears to be quite important. A therapeutic agent may be highly bioavailable but may not be bioactive, which may depend upon underlying disease process and biological status of myocardium, hence the efficacy could be enhanced by nutrient rich Mediterranean diet and mixture of nutraceuticals $[8,9,15]$. Beyond this therapy, Krasimira et al have reported a circadian twist in echocardiographic myocardial function, indicating that time adjusted therapy may be more potential in the management of HF because bioactivity of a therapy depends on circadian activity of the disease process [23].

In brief, it is possible that eating a prudent diet; fruits, vegetables, walnuts, legumes and flex seeds which are rich in micronutrients can be protective in patients with chronic heart failure. A package of micronutrient nutraceuticals, may also provide protection which needs further studies.

\section{CONFLICT OF INTEREST}

The authors confirm that this article has no conflict of interest.

\section{ACKNOWLEDGEMENTS}

Authors would like to acknowledgement International College of Nutrition for providing logistic support to write this article.

\section{REFERENCES}

[1] Witte KKA, Clark AL, Cleland JCF. Chronic heart failure and micronutrients. JACC 2001; 37: 1765-74.

[2] Lee JH, Jarreau T, Prasad A, Lavie C, O Keefe J, Ventura H. Nutritional assessment in heart failure patients. Cong Heart F 2011; 17: 199-203.

[3] Singh RB, Dube KP, Srivastav PK. Hypomagnesemia in relation to digoxin intoxication in children. Am Heart J 1976; 92: 144-7.
[4] Singh RB, Cameron EA. Relation of myocardial magnesium deficiency to sudden death in ischemic heart disease. Am Heart J 1982; 103: 899.

[5] Cameron EA, Singh RB. Magnesium and sudden death from heart disease. Lancet 1979; 2: 1084.

[6] Lombardi C, Carubelli V, Lazzarini V, et al. Effects of oral amino acid supplements on functional capacity in patients with chronic heart failure. Clin Med Insights: Cardiol 13, 2014.

[7] Kumar A, Singh RB, Saxena M, et al. Effect of carni Q-gel (ubiquinol and carnitine) on cytokines in patients with heart failure in the Tishcon study. Acta Cardiol 2007; 62: 349-354.

[8] Rothberg MB, Sivalingam SK. The New Heart Failure Diet: less salt restriction, more micronutrients. J Gen Intern Med 2010; 25; 1136-1137.

[9] Alsafwah S, Laguardia SP, Arroyo M, et al. Congestive heart failure is a systemic illness: a role for minerals and micronutrients. Clin Med Res 2007; 5: 238-43.

[10] Witte KK, Nikitin NP, Parker AC, et al. The effect of micronutrient supplementation on quality-of-life and left ventricular function in elderly patients with chronic heart failure. Eur Heart J 2005; 26: 2238-44. doi: 10.1093/eurheartj/ehi442.

[11] Mozaffarian D, Lemaitre RN, King IB, et al. Circulating longchain $\omega-3$ fatty acids and incidence of congestive heart failure in older adults: the cardiovascular health study: a cohort study. Ann Intern Med $2011 ; 155: 160-70$

[12] Pittler MH, Ernst E. Hawthorne extract for treating chronic heart failure; a metaanalysis of randomized trials. Am J Med 2003; 114 : 665-674.

[13] Hristova K, Singh RB, Fedacko J, et al. Causes and risk factors of congestive heart failure in India. World Heart J 2013; 5: 13-20.

[14] Fedacko J, Singh RB, Gupta A, et al. Inflammatory mediators in chronic heart failure in north India. Acta Cardiol 2014; 69: 391984.

[15] Abbate A. The heart at fire: inflammasome and cardiomyopathy. Exp Physiol 2013; 98: 385.

[16] Wang Y, Seto SW, Golledge J. Angiotensin II, sympathetic nerve activity and chronic heart failure. Heart Fail Rev 2014; 19: 187-98.

[17] Dai J, Lampert R, Wilson PW, Goldberg J, Ziegler TR, Vaccarino $\mathrm{V}$. Mediterranean dietary pattern is associated with improved cardiac autonomic function among middle-aged men: a twin study. Circ Cardiovasc Qual Outcomes 2010; 3(4): 366-73.

[18] Gal D, Pella D, Singh RB. The effect of an alpha-linolenic acid rich diet on the circadian rhythm of cardiac events. World Heart J 2008; 1: 49-56.

[19] Hristova K, Nakaoka T, Otsuka K, et al. Perspectives on chocolate consumption and risk of cardiovascular diseases and cognitive function. The Open Nutra J 2012; 5: 207-12.

[20] Singh RB, Rastogi SS, Verma R, et al. Randomized, controlled trial of cardioprotective diet in patients with recent acute myocardial infarction: results of one year follow up. BMJ 2002; 304: 1115-19.

[21] Singh RB, Dubnov G, Niaz MA, et al. Effect of an IndoMediterranean diet on progression of coronary disease in high risk patients: a randomized single blind trial. Lancet 2002; 360: 145561.

[22] John JV, McMurray MD, Packer M, et al . Angiotensin-neprilysin inhibition versus enalapril in heart failure. N Engl J Med 2014; 371: 993-1004.

[23] Krasimira H, Germaine C, Jan F, RB Singh. Echocardiographic study of circadian myocardial function among clinically healthy subjects. World Heart J 2014 (in press).

Received: December 05, 2014

Revised: December 20, 2014

Accepted: December 29, 2014

(C) Singh et al.; Licensee Bentham Open.

This is an open access article licensed under the terms of the Creative Commons Attribution Non-Commercial License (http://creativecommons.org/licenses/ by-nc/3.0/) which permits unrestricted, non-commercial use, distribution and reproduction in any medium, provided the work is properly cited. 